specifically crosschecked with a separate histopathology database. Univariate and subsequent multivariate analysis were carried out for biliary and vascular invasion individually and in combination with other prognostic variables to assess their clinical significance. Survival was assessed using Kaplan-Meier plots and log rank tests for significance $(p<0.05)$ using SPSS V.19.

Results 432 patients (67\% male, mean age 64.5 years, range $29-86$ ) underwent liver resection for CRLM during this time period. Primary tumours were either colonic (54\%) or rectal (46\%). Seventy patients $(16.3 \%)$ had positive biliary invasion on tumour histopathology whereas 137 (32\%) had vascular invasion. Overall 155 (36\%) patients had both biliary and vascular invasion (BVI). Overall 5 -year survival was $43 \%$ in this series. On univariate analysis 5 -year survival in those patients with biliary invasion was $39.9 \%$ compared to $42.5 \%$ in those without biliary invasion $(p=0.866)$. Results were similar in those patients with or without vascular invasion respectively $(40.4 \%$ vs $43.2 \%, \mathrm{p}=0.65)$. Also combined BVI failed to influence OS ( $39.2 \%$ vs $42.2 \%, p=0.856)$.

Conclusion In our series, bilary invasion of CRLM does not affect overall survival rates in patients having liver resection.

Competing interests None declared.

\section{PWE-147 COLORECTAL LIVER METASTASIS (CRLM): INCREASING ROLE OF LAPAROSCOPIC LIVER RESECTION-A SINGLE UNIT COMPARATIVE ANALYSIS}

doi:10.1136/gutjnl-2012-302514d.147

S K P John,* S Rehman, A Vallance, S Robinson, J French, D Manas, S White. Hepatobiliary Surgery, Newcastle Hospitals NHS Foundation Trust, Newcastle upon Tyne, UK

Introduction Laparoscopic liver resection (LLR) is becoming increasingly used to reduce the morbidity of open liver resection The aim of this study was to compare outcomes after LLR with that of open liver resection.

Methods From April 2007 onwards all patients who underwent either left lateral sectionectomy, left hemi-hepatectomy, segmentectomy and non-segmental resection for CRLM were identified from a prospectively maintained HPB database (open and LLR). Those having right hepatectomy were excluded from analysis as there were too few laparoscopic procedures for meaningful analysis. Comparisons between groups were made in terms of complications (graded using the Clavien-Dindo classification), duration of hospital stay and overall survival (OS). Statistical analysis was performed using Fisher Exact test for categorical variables, Mann-Whitney U test for non-parametric continuous variables and overall survival (OS) plotted with Kaplan-Meier curves (SPSS V.19).

Results 78 patients had LLR for various indications (colorectal $n=43$, non-colorectal $n=17$, benign $n=18$ ). During the same period 94 patients had open equivalent procedures for CRLM (including 4 conversions from a lap procedure). Female patients were more likely to have a LLR compared to open ( $47 \%$ females, $24 \%$ males $p=0.01$ ). Grade 3 and 4 complications were more seen in the open group (8.5\% vs $4.7 \%$ ), however grade 1 and 2 complications were slightly higher in the laparoscopic group (18.6\% vs $17.1 \%)$ Median stay was 4 days in LLR group (range 1-23), 7 days (range 3-95) in open group, $p<0.001$. $R 1$ resections were less during second era of the study in LLR and comparable to the open group. At 3 years $91 \%$ of LLR and $72 \%$ of open group were alive. OS was similar $(p=0.4)$.

Conclusion Laparoscopic liver resection has shown benefits in terms of lower morbidity in our series when comparing it to equivalent open procedures. Long term follow-up will be needed to see if there is real advantage in OS and outcome.

Competing interests None declared.

\section{PWE-148 BOUVERET'S SYNDROME: LEAVING NO STONE UNTURNED}

doi:10.1136/gutjnl-2012-302514d.148

${ }^{1} \mathrm{~S}$ Fong, ${ }^{*}{ }^{1} \mathrm{E}$ Oderuth, ${ }^{2} \mathrm{~F}$ Langlands, ${ }^{1} \mathrm{~A}$ Riding, ${ }^{1} \mathrm{~J}$ Rademaker. ${ }^{1}$ Department of Gastroenterology, Conquest Hospital, Hastings, UK; ${ }^{2}$ Department of Surgery, Conquest Hospital, Hastings, UK

Introduction Bouveret's syndrome is a clinically distinct form of gallstone ileus caused by the formation of a fistula between the biliary tract and duodenum. Early recognition and treatment can significantly improve morbidity and mortality. We present two cases that have been successfully treated at our hospital.

Methods Patient A is 71-year-old lady who was referred for gastroscopy after a CT abdomen that was performed to investigate her weight loss showed deformity of the duodenal cap. The gastroscopy showed a large gallstone obstructing the pyloric opening (Abstract PWE-148 figure 1A). Endoscopic removal was unsuccessful. She then developed vomiting and abdominal pain. Blood tests showed raised inflammatory markers and liver function tests. Repeat gastroscopy showed that the gallstone had passed down the pylorus, revealing a fistula opening in the duodenum (Abstract PWE-148 figure 1B). Diagnosis was confirmed on repeat CT abdomen which showed pneumobilia and two large gallstones that had migrated to the ileum (Abstract PWE-148 figure 1C). Patient B is 87-year-old lady who presented with a 1-week history of vomiting and haematemesis. Her blood tests on admission showed raised inflammatory markers with normal liver function tests. Gastroscopy showed oesophagitis, a duodenal ulcer and obstructing gallstone. Gastrograffin studies then showed that the gallstone had migrated to the proximal jejunum (Abstract PWE-148 figure 1D) and this was confirmed on CT abdomen.

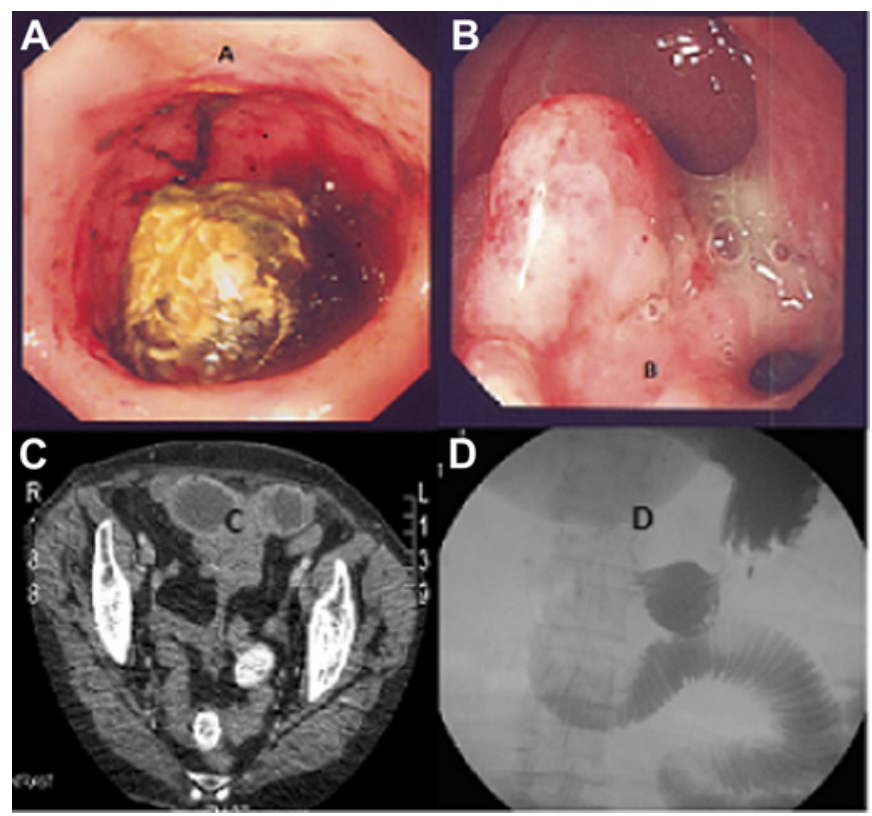

Abstract PWE-148 Figure 1

Results Patient A was treated with a Ryle's tube insertion for stomach decompression, intravenous fluids, antibiotics and peripheral parenteral nutrition. She underwent a laparotomy, which showed distended fluid filled small bowel, with a large $5 \times 3 \mathrm{~cm}$ gallstone lodged in the ileum. An ileal enterotomy was performed and the gall stone excised. Patient B was treated with a Ryle's tube insertion, intravenous fluids and peripheral parenteral nutrition. She underwent a laparotomy, in which a gallstone was found lodged in 
the proximal jejunum and surgically removed. Both patients made a full recovery and were discharged home.

Conclusion Bouveret's syndrome is rare and accounts for $1-3 \%$ of gallstone ileus. We have now encountered two cases in our hospital recently. This may reflect the rise in our ageing population, as a major risk factor for developing this syndrome is age $>70$ years. Establishing the diagnosis early requires an awareness of this syndrome and prompt recognition of the signs. Contrast enhanced $\mathrm{CT}$ is the investigation of choice. In addition to this, a combined care approach between the gastroenterologists, surgeons and nutrition team is crucial to a successful outcome, hence our wish to present these cases.

Competing interests None declared.

\section{REFERENCE}

1. Cappell MS, Davis M. Characterization of Bouveret's syndrome: a comprehensive review of 128 cases. Am J Gastroenterology 2006;101:2139-46.

\section{PWE-149 GENE EXPRESSION IN OXALIPLATIN RELATED SINUSOIDAL OBSTRUCTION SYNDROME}

doi:10.1136/gutjnl-2012-302514d.149

${ }^{1,2} \mathrm{~S}$ Robinson, ${ }^{1} \mathrm{~J}$ Mann, ${ }^{2} \mathrm{D}$ Manas, ${ }^{1} \mathrm{D}$ Mann, ${ }^{2} \mathrm{~S}$ White. ${ }^{1}$ Institute of Cellular Medicine, Newcastle University, Newcastle upon Tyne, UK; ${ }^{2} H P B$ \& Transplant Surgery, Freeman Hospital, Newcastle upon Tyne, UK

Introduction Sinusoidal Obstruction Syndrome (SOS) is associated with Oxaliplatin based chemotherapy in patients with colorectal liver metastases (CRLM) and is a cause of concern when undertaking major liver resection. The pathogenesis of SOS is poorly understood however a variety of candidate genes have been identified which may play a role in activating various molecular pathways involved. The aim of this study was to validate these gene expression changes in an independent cohort of patients.

Methods Patients undergoing liver resection for CRLM, were identified for inclusion and appropriate informed consent obtained. Full clinical information was recorded for each patient including preoperative chemotherapy use. A biopsy of the non-tumour bearing liver was obtained, prior to parenchymal transection, and stored in RNAlater. Histopathology was reviewed to identify those with SOS. Hepatic gene expression was compared in chemotherapy naive patients (controls) with no evidence of underlying liver disease $(\mathrm{n}=10)$ and those who received pre-operative Oxaliplatin either with $(n=13)$ or without $(n=9)$ evidence of SOS by qRT-PCR. Mann-Whitney $U$ test was used to assess statistical significance.

Results The interval between cessation of chemotherapy and surgery was similar for all patient groups $(p=0.45)$. In contrast to previous studies we were unable to identify changes in extracellular matrix remodelling genes (MMP2, MMP9, TIMP1, TGF $\beta$ ) thought to be involved in SOS. However there was up regulation of angiogenesis related VEGF-C (1.6-fold, $p<0.05)$ along with the hypoxia induced HIF1 $\alpha(1.98$-fold; $\mathrm{p}<0.01)$ in those with SOS. It is suggested that SOS is associated with a pro-thrombotic tendency and in keeping with this there was a non-significant trend towards increased expression of vWF (2.5-fold; $p=0.06)$. We also confirmed up-regulation of CCL20 in those with SOS (3.8-fold; $p<0.05)$ which is chemotactic for colorectal cancer cells.

Conclusion We were able to confirm up-regulation of genes involved in angiogenesis, hypoxia and thrombogenesis in patients with SOS. It is likely that changes in extracellular matrix re-modelling genes occur early in the development of SOS and but have returned to baseline levels if there is a reasonable duration between stopping chemotherapy and surgical resection. Increased expression of CCL20 may account for the recently reported poorer disease specific survival in patients with SOS.

Competing interests None declared.

\section{PWE-150 CHEMOTHERAPY ASSOCIATED LIVER INJURY: A SYSTEMATIC REVIEW AND META-ANALYSIS}

doi:10.1136/gutjnl-2012-302514d.150

${ }^{1,2} \mathrm{~S}$ Robinson, ${ }^{2} \mathrm{C}$ Wilson, ${ }^{1} \mathrm{~A}$ Burt, ${ }^{2} \mathrm{D}$ Manas, ${ }^{2} \mathrm{~S}$ White. ${ }^{1}$ Institute of Cellular Medicine, Newcastle University, Newcastle upon Tyne, UK; ${ }^{2}$ HPB \& Transplant Surgery, Freeman Hospital, Newcastle upon Tyne, UK

Introduction Chemotherapy associated liver injury (CALI) has been associated with increased morbidity and mortality in patients undergoing major hepatectomy to treat colorectal liver metastases (CRLM). In addition a link has recently been made between CALI and poorer long term disease specific outcome. The aim of this review was to determine the pathological effect of specific chemotherapy regimens on the hepatic parenchyma as well as surgical morbidity, mortality and overall survival.

Methods A literature search of MEDLINE, EMBASE and the Cochrane Library identified 14619 potentially relevant reports. Of these 37 full text reports which included patients only with CRLM and provided either histological data or patient outcome data were considered suitable for inclusion in this review. For each report data relating to study design characteristics, histological scoring of the liver parenchyma and peri-operative outcomes were extracted using a standardised proforma. Study quality was assessed using the Newcastle-Ottawa score for non-randomised studies and the grade of evidence assessed according to the Oxford centre for Evidence Based Medicine scale. A meta-analysis was performed utilising the random effects model of DerSimmonian and Laird. Results are reported as $R R( \pm 95 \% \mathrm{CI}$ ). Statistical significance was set at $p<0.05$. Results No association could be demonstrated between the use of pre-operative chemotherapy and the development of hepatic steatosis $>30 \%$. The presence of steatohepatitis was associated with the use of pre-operative Irinotecan based chemotherapy (RR 3.45; 95\% CI 1.12 to $10.62 ; p=0.03)$. Calculating the number needed to harm suggests that one in every 12 patients treated with Irinotecan based chemotherapy could be expected to develop steatohepatitis. Oxaliplatin based chemotherapy regimens are associated with grade 2 or greater sinusoidal injury (RR 4.36; 95\% CI 1.12 to $10.62 ; p=0.03$ ) with a number needed to harm of 8 . The use of Bevacizumab alongside Oxaliplatin reduces the risk of grade 2 or greater sinusoidal injury.

Conclusion The use of pre-operative chemotherapy is associated with an increased risk of injury to the liver parenchyma in patients with CRLM. This injury occurs in a regimen specific manner with Irinotecan being associated with steatohepatitis whereas Oxaliplatin is associated with sinusoidal injury. This injury may have implications on the functional reserve of the liver following major hepatic resection.

Competing interests None declared.

\section{PWE-151 EMERGENCY ERCP IN CRITICALLY ILL PATIENTS IS A SUCCESSFUL PROCEDURE}

doi:10.1136/gutjnl-2012-302514d.151

V P K Lekharaju, ${ }^{*}$ J Iqbal, 0 Noorullah, N Polavarapu, S Menon, S Hood, N Stern, R Sturgess. Digestive Diseases Unit, Aintree University Hospital NHS Trust, Liverpool, UK

Introduction Emergency ERCP may be required in patients with biliary sepsis who rapidly deteriorate with multi-organ dysfunction and cannot wait until the next available list. The majority of these patients require ventilatory and/or inotropic support and general anaesthesia for stabilisation. The data on the outcome of emergency ERCP in this patient cohort is limited. We sought to assess the frequency, indications, and clinical outcomes of emergency ERCPs. 